\title{
Model-based Characterization of Mammographic Masses
}

\author{
Sven-René von der Heidt ${ }^{1}$, Matthias Elter ${ }^{2}$, Thomas Wittenberg ${ }^{2}$, \\ Dietrich Paulus ${ }^{1}$ \\ ${ }^{1}$ Institut für Computervisualistik, \\ Universität Koblenz-Landau \\ ${ }^{2}$ Abteilung Bildverarbeitung und Medizintechnik, \\ Fraunhofer-Institut für Integrierte Schaltungen \\ agas@uni-koblenz.de
}

\begin{abstract}
The discrimination of benign and malignant types of mammographic masses is a major challenge for radiologists. The classic eigenfaces method was recently adapted for the detection of masses in mammograms. In the work at hand we investigate if this method is also suited for the problem of distinguishing benign and malignant types of this mammographic lesion. We furthermore evaluate two extended versions of the eigenfaces approach (fisherface and eigenfeature regularization extraction) and compare the performance of all three methods on a public mammography database. Our results indicate that all three methods can be applied to discriminate benign and malignant types of mammographic masses. However, our ROC analysis shows that the methods still require combination with other features to allow for reliable classification.
\end{abstract}

\section{Introduction}

Mammography is the most effective technique for the early detection and diagnosis of breast cancer available today. One of the most difficult tasks in the context of mammogram interpretation is the discrimination of benign and malignant mass lesions, which are defined as space-occupying lesions that can be seen in at least two different projections [1]. Recently, Oliver et al. [2] have proposed to apply the classic eigenface method to a related task, the detection of mammographic masses. Their results indicate that the eigenface method is a promising approach to the detection of masses, or more precisely, the discrimination of normal tissue and mammographic masses. In the work at hand, we investigate if the eigenface method is also suited to discriminate between benign and malignant types of mammographic masses. Furthermore, we have implemented two extended versions of the eigenface method: the fisherface and the eigenfeature-regularization-extraction methods. We compare the receiver operating characteristic curve performance of all three approaches on a public mammography database. There has been considerable interest in the classification of mammographic masses in the last decade. Due to the broad variety of approaches that have been published in the last years, we can mention only 
some representative examples of state of the art methods here. In contrast to the three approaches that we propose in this work, most of the work that can be found in literature follows the classic pattern recognition chain: segmentation of a mass from the background tissue, feature extraction and classification. Good examples are the work of Shi et al. [3] who apply level-sets for the segmentation of masses and propose several features specifically designed for the task of mass characterization. Varela et al. [4] have developed features that measure the degree of sharpness and microlobulation of the mass margin to improve their previously proposed CAD approach. Drukker et al. [5] extract mass features from both mammograms and ultrasound images and thus have proposed a promising multi-modal approach for the discrimination of masses. Timp and Karssemeijer [6] have developed a segmentation method based on dynamic programming to improve the classification performance of their previously proposed system for mass characterization.

The rest of this work is organized as follows: in Sec. 2 the eigenface, fischerface and eigenfeature regularization extraction methods (ERE method) are briefly described. Furthermore, details on the database of mammograms that we use to develop and evaluate the proposed approach are provided. In Sec. 3 the experiments and their results that we have performed to evaluate the classification performance of the proposed approaches are described. Finally, we close this work with a discussion of our results and provide possible directions for future research (Sec. 4).

\section{Materials and methods}

We use the digital database for screening mammography (DDSM) [7] for our experiments. It is a widely used public reference database for computer-aided detection and diagnosis of mammographic lesions. For this study we have randomly selected 206 regions of interest (ROIs) containing mammographic masses from the DDSM. 114 ROIs contained malignant and 92 ROIs benign masses. In the following we will apply eigenface techniques to the input images. This requires that each image has the same size. We crop ROIs from the mammograms and normalize them to a standard size. Another major problem are the different intensity ranges for the ROIs. Hence, we rescale each region to a normalized distribution of gray-levels. The principal component analysis (PCA) provides the basis for the following mathematical approaches. It is also called the Karhunen-Loeve transform (KLT) [8].

- The eigenface method was introduced by Turk and Pentland [9] for face recognition. It requires a set of images that have each the same size. For faces the method works best if the images are aligned (e.g. the nose is in the center of each image). Eigenfaces (or eigenvectors) are obtained from the covariance matrix of the probability distribution of the vector space of possible faces (in our case mammographic masses) using the PCA.

- Another approach used in face recognition is due to Fisher [10]. It extends the eigenface method by a LDA (linear discriminant analysis). The input 
images are divided into subsets for each class. Two covariance matrices are computed for intra-class features and inter-class distances. A linear transform is computed that simultaneously de-correlates the feature vectors and class separation. The fisherface method is known to be less sensitive to variation in lighting and pose of the object than the eigenface method.

- The ERE method [11] further extends the eigenface method and particularly inspects those eigenvectors who's eigenvalues are zero.

\section{Results}

For the experiments we normalize and pre-process the input images as outlined in Sect. 2. We have evaluated and compared the classification performance of the three proposed approaches using 10-fold cross validation and receiver operating characteristic (ROC) curve analysis. We use the area under the ROC curve $A_{z}$ as a performance metric. The classification is done using the $k$-nearest neighbors $(\mathrm{kNN})$ classifier with Euclidean distance metric in eigenfeature space. We use the program ROCKIT by Charles E. Metz, PhD, to determine $A_{z}$ based on the output of the ckNN classifier [12]. Fig. 1 shows ROC-curves for eigen- and fisherfaces and the ERE method. Fig. 1 shows results for the DDSM-database with uniform size for input images of $80 \times 80$ pixels. The training set for all tests consisted of 206 ROIs. The $A_{z}$ values are 0.5256 for the Fisherfaces, 0.5617 for

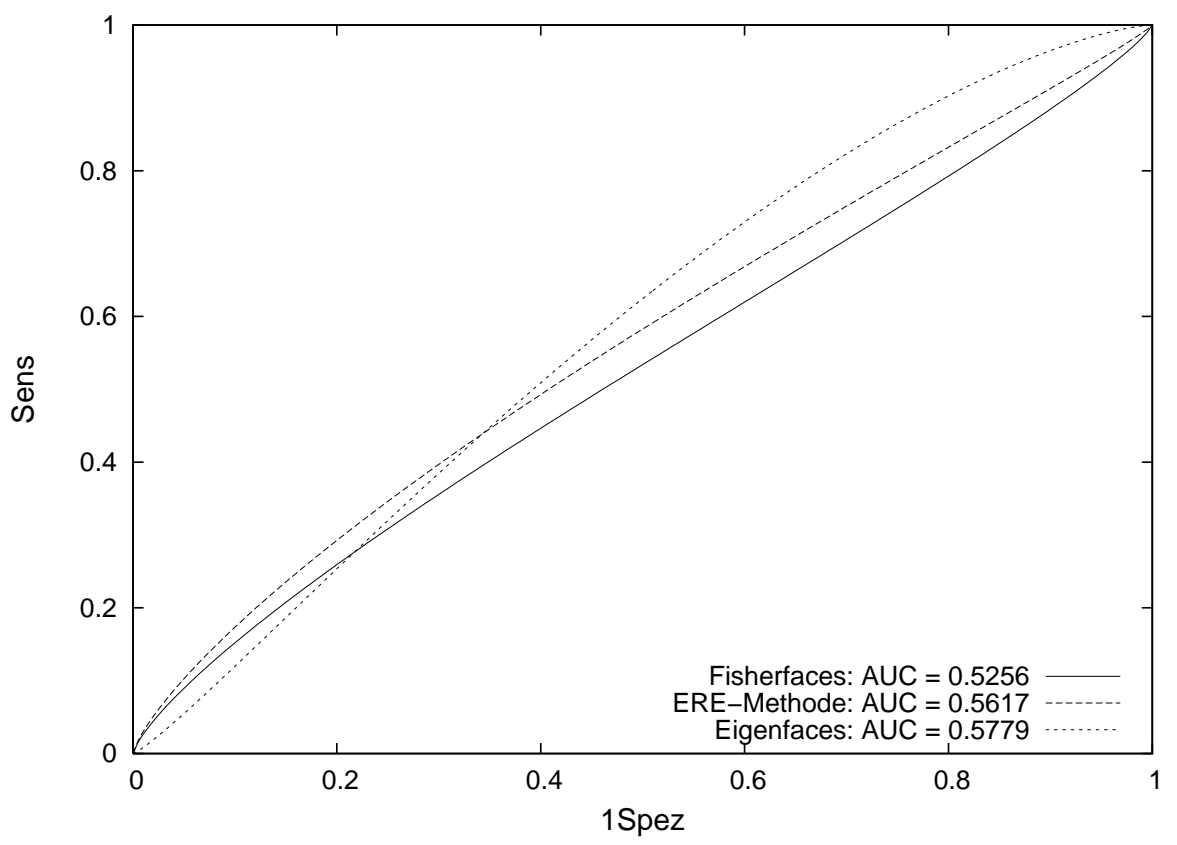

Fig. 1. Tests with uniform scaling to $80 \times 80$ pixel using 206 ROIs 
Table 1. Results with uniform scaling to $80 \times 80$ pixel and 206 ROIs for different numbers of eigenfeatures.

\begin{tabular}{cccc}
\hline \multirow{2}{*}{ Number of eigenfeatures } & \multicolumn{3}{c}{ AUC values } \\
\cline { 2 - 4 } & Eigenfaces & Fisherfaces & ERE method \\
\hline 10 & 0.6017 & 0.5623 & 0.5496 \\
all & 0.6017 & 0.5161 & 0.5559 \\
& 0.5941 & 0.5155 & 0.5406 \\
\hline
\end{tabular}

Table 2. Results with grouping by ROI size instead of scaling and 206 ROIs for different numbers of eigenfeatures.

\begin{tabular}{cccc}
\hline \multirow{2}{*}{ Number of eigenfeatures } & \multicolumn{3}{c}{ AUC values } \\
\cline { 2 - 4 } & Eigenfaces & Fisherfaces & ERE method \\
\hline 10 & 0.5916 & 0.5152 & 0.5713 \\
40 & 0.6000 & 0.5016 & 0.5735 \\
all & 0.5988 & 0.5527 & 0.5422 \\
\hline
\end{tabular}

the ERE-Method, and 0.5779 for eigenfaces. Table 1 and Table 2 summarize AUC results for different experiments. For Table 2 we grouped the ROIs of similar sizes and avoided resizing.

\section{Discussion}

We have proposed to apply the eigenface method to the discrimination of benign and malignant mass. We showed that the eigenface method and two extended approaches can be used for discriminating benign and malignant mammographic masses. However, the AUC values show that the methods are not reliable enough, yet, for diagnosis. Interestingly, the classic eigenface method seems to be better suited for mass classification than the two extended approaches, which are known to be superior in other applications [10,11]. We tested different preprocessing approaches and came to the result that scaling as required for eigenfaces actually reduces the classification performance. We are confident that manual selection of the examples used for the estimation of the covariance matrices will yield better values.

Acknowledgement. The authors are also grateful to Charles E. Metz, PhD, for providing the ROCKIT program for ROC analysis. This work was supported in part by the Bayerische Forschungsstiftung within the scope of the project Mammo-iCAD.

\section{References}

1. American College of Radiology. Breast Imaging Reporting and Data System BIRADS Atlas; 2006. 
2. Oliver A, Marti J, Bosch A, et al. A new approach to the classification of mammographic masses and normal breast tissue. In: The 18th International Conference on Pattern Recognition (ICPR'06); 2006. p. 707-710.

3. Shi J, Sahiner B, Chan HP, et al. Characterization of mammographic masses based on level set segmentation with new image features and patient information. Med Phys. 2008;35(1):280-290.

4. Varela C, Timp S, Karssemeijer N. Use of border information in the classification of mammographic masses. Phys Med Biol. 2006;51(2):425-441. Available from: http://dx.doi.org/10.1088/0031-9155/51/2/016.

5. Drukker K, Horsch K, Giger ML. Multimodality computerized diagnosis of breast lesions using mammography and sonography. Acad Radiol. 2005;12(8):970-979. Available from: http://dx.doi.org/10.1016/j.acra.2005.04.014.

6. Timp S, Karssemeijer N. A new 2D segmentation method based on dynamic programming applied to computer aided detection in mammography. Med Phys. 2004;31(5):958-971.

7. Heath M, Bowyer K, Kopans D, et al. The digital database for screening mammography. Procs Int Workshop on Digital Mammography. 2001; p. 212-218.

8. Kessler W. Multivariante Datenanalyse: Für die Pharma-, Bio- und Prozessanalyse. WILEY-VCH Verlag GmbH \& Co. KGaA, Weinheim; 2007.

9. Turk M, Pentland A. Eigenfaces for Recognition. J Cognitive Neuroscience. 1991;3(1):71-86.

10. Belhumeur PN, Hespanha JP, Kriegman DJ. Eigenfaces vs. fisherfaces: Recognition using class specific linear projection. IEEE Trans PAMI. 1997;19(7):711-720.

11. Jiang X, Mandal B, Kot A. Eigenfeature regularization and extraction in face recognition. IEEE Trans PAMI. 2008;30(3):383-394.

12. Metz CE, Herman BA, Shen JH. Maximum-likelihood estimation of ROC curves from continuously-distributed data. Stat Med. 1998;17:1033-1053. 